\title{
Humanized mouse model of Rasmussen's encephalitis supports the immune-mediated hypothesis
}

\author{
Hania Kebir, ${ }^{1,2}$ Lionel Carmant, ${ }^{3,4,5}$ François Fontaine, ${ }^{3,4}$ Kathie Béland, ${ }^{3}$ Ciprian M. Bosoi, ${ }^{3}$ Nathalie T. Sanon, ${ }^{3}$ Jorge I. Alvarez, ${ }^{6}$ \\ Sébastien Desgent, ${ }^{3}$ Camille L. Pittet, ${ }^{2}$ David Hébert, ${ }^{3}$ Marie-Josée Langlois, ${ }^{3}$ Rose-Marie Rébillard, ${ }^{4}$ Dang K. Nguyen, ${ }^{2,5}$ \\ Cécile Cieuta-Walti, ${ }^{7}$ Gregory L. Holmes, ${ }^{8}$ Howard P. Goodkin, ${ }^{9}$ John R. Mytinger, ${ }^{9}$ Mary B. Connolly, ${ }^{10}$ Alexandre Prat, ${ }^{2,5}$ \\ and Elie Haddad ${ }^{1,3,4}$ \\ 'Department of Microbiology, Infectious Diseases, and Immunology, Faculty of Medicine, Université de Montréal, Montreal, Quebec, Canada. 'Centre de Recherche du Centre Hospitalier de I'Université \\ de Montréal (CRCHUM), Faculty of Medicine, Université de Montréal, Montreal, Quebec, Canada. ${ }^{3}$ Centre de Recherche du Centre Hospitalier Universitaire Sainte-Justine (CHU Sainte-Justine), Montreal, \\ Quebec, Canada. ${ }^{4}$ Department of Pediatrics, and ${ }^{5}$ Department of Neurosciences, Faculty of Medicine, Université de Montréal, Montreal, Quebec, Canada. ${ }^{6}$ Department of Pathobiology, School of Veterinary \\ Medicine, University of Pennsylvania, Philadelphia, Pennsylvania, USA. 'Tentre Hospitalier Universitaire de Sherbrooke, Department of Pediatrics, Université de Sherbrooke, Shebrooke, Quebec, Canada. \\ ${ }^{8}$ Department of Neurological Sciences, Larner College of Medicine, University of Vermont, Burlington, Vermont, USA. ${ }^{9}$ Fontaine Research Park, Department of Pediatrics, University of Virginia, Charlottesville, \\ Virginia, USA. ${ }^{10}$ B.C. Children's Hospital, Division of Neurology, Department of Pediatrics, University of British Columbia, Vancouver, British Columbia, Canada.
}

\begin{abstract}
Rasmussen's encephalitis (RE) is a chronic inflammatory brain disorder that causes frequent seizures and unilateral hemispheric atrophy with progressive neurological deficits. Hemispherectomy remains the only treatment that leads to seizure freedom for this refractory epileptic syndrome. The absence of an animal model of disease has been a major obstacle hampering the development of effective therapies. Here, we describe an experimental mouse model that shares several clinical and pathological features with the human disease. Immunodeficient mice injected with peripheral blood mononuclear cells from RE patients and monitored by video electroencephalography developed severe seizures of cortical origin and showed intense astrogliosis and accumulation of human IFN- $\gamma$ - and granzyme B-expressing T lymphocytes in the brain compared with mice injected with immune cells from control subjects. We also provide evidence for the efficacy of $\alpha 4$ integrin blockade, an approved therapy for the treatment of multiple sclerosis and Crohn's disease, in reducing inflammatory markers associated with RE in the CNS. This model holds promise as a valuable tool for understanding the pathology of RE and for developing patient-tailored experimental therapeutics.
\end{abstract}

\section{Introduction}

Rasmussen's encephalitis (RE) is a severe brain disorder that usually manifests itself during childhood with the onset of frequent focal seizures (1). These seizures often progress to epilepsia partialis continua (EPC), a state of near-continuous seizures that tend to be highly refractory to standard antiepileptic drugs (2). In most cases, the only treatment option to achieve seizure freedom is a hemispherectomy to functionally disconnect or completely remove the affected side of the brain (3). With the assumption that $\mathrm{RE}$ is an immune-mediated disorder, a variety of immunomodulatory therapies have been proposed to slow down disease progression but have yielded conflicting results, especially in the long term. These include corticosteroids, intravenous immunoglobulins (IVIGs), tacrolimus, and the B cell-depleting CD20 antibody rituximab (4-9).

\section{Related Commentary: p. 1724}

Authorship note: HK and LC contributed equally to this work. AP and EH are co-senior authors.

Conflict of interest: The authors have declared that no conflict of interest exists.

Submitted: August 29, 2017; Accepted: February 13, 2018.

Reference information: J Clin Invest. 2018;128(5):2000-2009.

https://doi.org/10.1172/JC197098.
A fundamental challenge in developing new treatment strategies to treat $\mathrm{RE}$ is that relatively little is known about the etiology and pathophysiology of the disease. Although viral (1,10-14), autoimmune, and antibody-mediated (15-22) etiologies have been suspected, none was ever effectively confirmed. The most consistent histopathological feature of RE is a CNS-targeted inflammation characterized by $\mathrm{T}$ lymphocyte infiltration and microglial nodules, which are associated with substantial neuronal loss leading to neuronophagia (23) and gliosis $(24,25)$. A growing body of evidence now points to the crucial role of $\mathrm{CD} 8^{+} \mathrm{T}$ lymphocytes in $\mathrm{RE}$ pathogenesis $(26,27)$.

Another major limitation that has made it difficult to evaluate the effectiveness of alternative treatment options for patients with $\mathrm{RE}$ is the lack of a relevant experimental model of disease (11, 17, $20,22)$. The rarity of the condition further complicates the testing of potential therapies in clinical trials. To overcome this and allow individualized screening of new therapeutics to treat $\mathrm{RE}$ and better understand its pathophysiology, we generated an experimental model of the disease by engrafting human peripheral blood mononuclear cells (PBMCs) obtained from RE patients into NOD/ LtSz-scid IL-2R $\gamma$ c(null) (NSG) mice. These severely immunodeficient mice are fast becoming instrumental investigative tools in translational biomedical research, particularly because they allow study of the human immune response in autoimmune and inflam- 
matory diseases and facilitate the testing of therapies in an in vivo setting that is more relevant to human immunity $(28,29)$. This is in part owing to a complete null mutation in the IL-2 receptor common $\gamma$ chain (IL-2R $\gamma \mathrm{c}^{\text {null }}$ ), which enables NSG mice to tolerate the engraftment of human immune cells. Here, we postulated that the injection of RE patients' peripheral immune cells into NSG mice would generate a more personalized in vivo model that closely reflects characteristics of the human disease, therefore maximizing its value for translational research and preclinical studies.

\section{Results}

Engraftment of human PBMCs from RE patients induces seizures in NSG mice. For each control subject and RE patient (Supplemental Table 1; supplemental material available online with this article; https://doi.org/10.1172/JCI97098DS1), a minimum of 4 mice were injected i.p. with $1 \times 10^{6}$ PBMCs. FACS analysis showed successful engraftment of human cells from both the RE and control donors and revealed that the predominant cell type detected in the peripheral blood and spleens of the engrafted NSG mice were human $\mathrm{CD}^{+} \mathrm{T}$ lymphocytes (Figure 1A). Five of nineteen recipient animals, all injected with PBMCs from patient 3, died within a week of the procedure. All of the other animals tolerated the procedure well.

Sixteen of the nineteen (84\%) NSG mice engrafted with PBMCs from RE patients (RE-NSG) had multiple seizures, as documented by video electroencephalogram (EEG) recordings (Supplemental Video 1), and all had interictal EEG abnormalities (Supplemental Table 2). The seizures started around day 21 and were characterized by episodes of freezing, myoclonus, clonus, or rearing and falling, with an electrographic cortical onset that occasionally spread to limbic structures (Figure 1B). Seizures recurred on a daily basis for the first week and then progressively decreased to weekly seizures by week 8 . In contrast, none of the mice injected with cells from healthy donors or patients with non-RE-related temporal lobe epilepsy (control-NSG) experienced seizures, even with transfers of up to $10 \times 10^{6}$ human PBMCs (Figure $1 \mathrm{C}$ and Supplemental Table 2 ), which, however, caused disseminated xenograft versus host disease (xeno-GVHD), as previously reported (30).

To rule out the possibility that the disease was induced by viral transfer, 8 NSG mice were engrafted with irradiated PBMCs from 2 patients with RE. These animals failed to develop seizures or pathological signs of RE, whereas injection of nonirradiated PBMCs from the same RE patients elicited repetitive seizures in mice. Likewise, injection of plasma from RE patients did not cause seizures in the recipient NSG animals. Finally, no immunopositive signal for heat shock protein 73 (HSP73), normally suggestive of stress responses associated with viral infections, was found upon histopathological examination of CNS material from RE-NSG mice. Because viruses, which are usually resistant to radiation, were not present in the CNS of affected animals, blood-borne viral infections seem an unlikely direct cause of seizures in this model. This experiment further demonstrates that the induction of seizures in our model requires donor T lymphocyte proliferation.

Human proinflammatory $T$ lymphocytes expressing $I F N-\gamma$ and granzyme B infiltrate the brains of RE-NSG mice. We next sought to evaluate by FACS and microscopy the composition of the immune cell infiltrates within the CNS of recipient animals. We detected a significantly higher number of $\mathrm{CD} 45^{+}$mononuclear cells in the brains of RE-NSG mice compared with the brains of control-NSG mice over the 5-week observation period (Figure 1D and Supplemental Figure 1). Upon closer examination, we found that the number of human $\mathrm{CD} 45^{+}\left(\mathrm{hCD} 45^{+}\right)$leukocytes and human $\mathrm{CD} 4^{+}$ (hCD4 ${ }^{+}$) and $\mathrm{CD}^{+}\left(\mathrm{hCD}^{+}\right) \mathrm{T}$ lymphocytes infiltrating the CNS of recipient animals increased steadily over time during the 5-week period following PBMC transfer and was consistently higher in the brains of RE-NSG mice than in those of mice engrafted with control PBMCs. In contrast to this sharp increase in the number of CNS-infiltrating human cells, the absolute number of mouse $\mathrm{CD}_{4} 5^{+}\left(\mathrm{mCD} 45^{+}\right)$cells detected in the brains of RE-NSG mice peaked at week 2 after PBMC transfer, declined thereafter, and did not vary significantly from what was observed in the controlNSG mice, suggesting that the disease is mediated by the recruitment of hCD $45^{+}$rather than entry of $\mathrm{mCD} 45^{+}$cells into the brain (Figure 1D). Interestingly, we noted comparable frequencies of $\mathrm{hCD}^{+}$and hCD4 $4^{+} \mathrm{T}$ lymphocytes in the brains of RE-NSG mice at week 5 after PBMC transfer, a point at which leukocyte infiltration into the brain had reached its maximum (Figure 1D and Figure 2, A and B).

To investigate the role of inflammatory $\mathrm{T}$ cells in the disease process, we characterized $\mathrm{hCD}^{+}$and $\mathrm{hCD}^{+} \mathrm{T}$ lymphocytes in terms of their cytokine expression profile. FACS analyses revealed a significantly higher number of IFN- $\gamma$ - and IL-17-expressing hCD $4^{+}$ and hCD ${ }^{+} \mathrm{T}$ lymphocytes in the brains of RE-NSG animals than in the brains of control-NSG mice. These effector cells were detectable as soon as 2 weeks after PBMC transfer (Figure 2, C and D), with IFN- $\gamma$-producing T lymphocytes clearly outnumbering IL-17expressing cells. Whereas comparable numbers of $\mathrm{hCD}^{+}$and hCD $4^{+}$T lymphocytes expressed IFN- $\gamma$ in the CNS of RE-NSG mice, $\mathrm{hCD} 4^{+}$, rather than hCD8 ${ }^{+}, \mathrm{T}$ lymphocytes were the major source of IL-17 in the brain. We also evaluated the expression of granzyme B as a marker of cytotoxic activity and detected numerous granzyme $\mathrm{B}^{+} \mathrm{CD}^{+} \mathrm{T}$ lymphocytes in the brains of NSG mice (Figure $2 \mathrm{E}$ ). These findings are consistent with reports on the histopathological examination of brain specimens from patients with $\operatorname{RE}(23,24,26)$.

We did not detect human $\mathrm{CD} 19^{+}$B lymphocytes but did detect numerous HLA-DR-reactive cells, which were presumably human infiltrating antigen-presenting cells or myeloid hCD11c cells within the CNS of RE-NSG mice (Figure 2F and Supplemental Figure 2). Therefore, these findings identify IFN- $\gamma$ - and granzyme B-expressing T lymphocytes as well as HLA-DR ${ }^{+}$cells as major contributors to CNS inflammation and to the development of seizures in our model.

Astrogliosis and focal neuronal damage in the brains of RE-NSG mice. In terms of damage to the CNS, the brains of RE-NSG mice showed marked and strong immunostaining for glial fibrillary acidic protein (GFAP), which is indicative of astrogliosis (Figure 3, A and $\mathrm{B})$. We observed the most intense signal for GFAP in perivascular and submeningeal areas and diffused signal in proximal areas.

Another prominent pathological feature of RE is the presence of activated microglial cell aggregates called nodules, which may or may not be associated with degenerating neurons (2). Thus, to assess microglial activation in the brains of NSG mice, we stained brain tissue sections with the microglia/macrophage-specific marker ionized calcium-binding adaptor molecule 1 (Iba-1). 
A
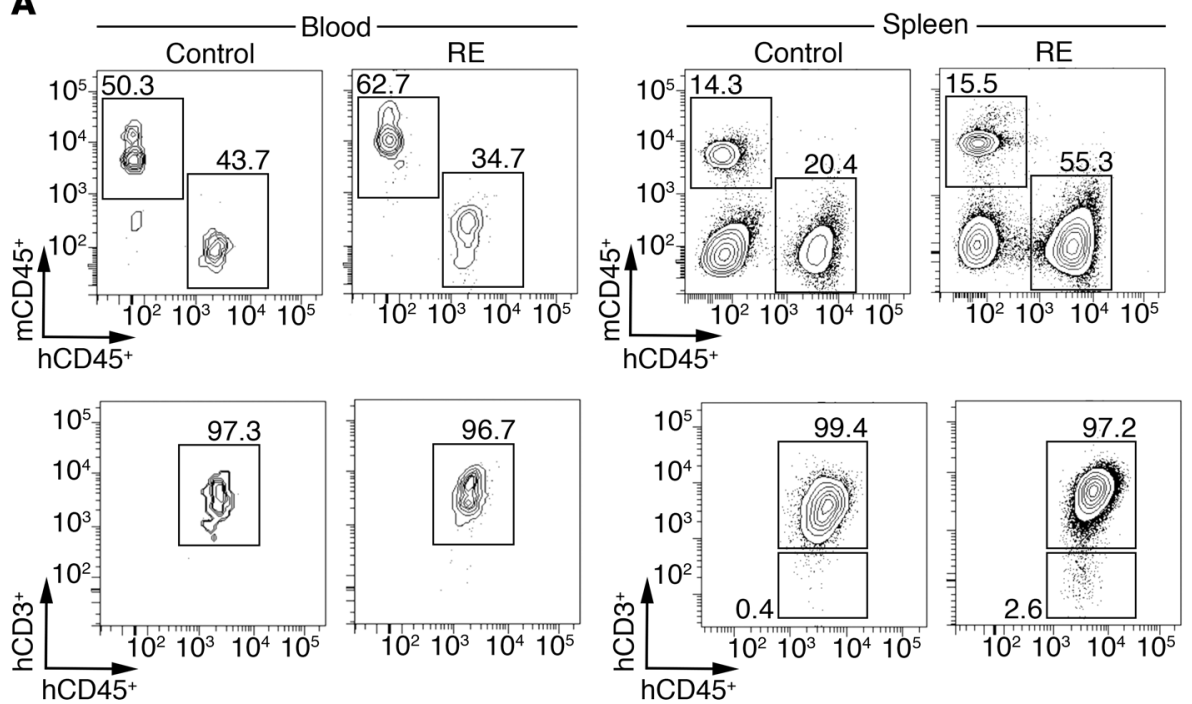

B
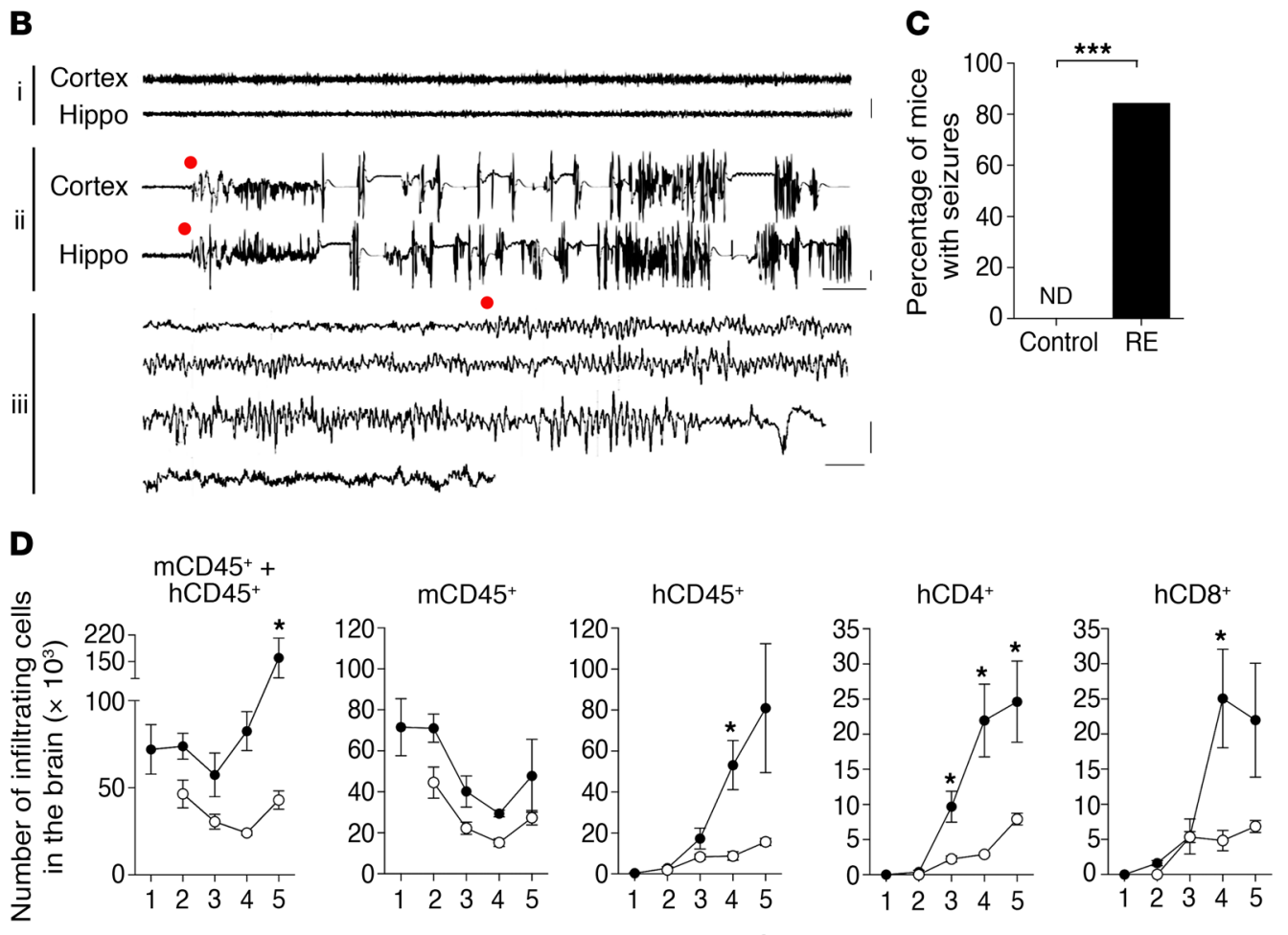

Weeks after PBMC transfer
Figure 1. Clinical and immunological profile of NSG mice engrafted with human control or RE PBMCs.

(A) FACS analyses of hCD45', $\mathrm{mCD} 45^{+}$, and $\mathrm{hCD}^{+}$cells in the blood and spleens of NSC animals injected with PBMCs from RE patients or controls, 2 weeks after transfer. (B) Representative EEG recordings from an NSG animal engrafted with (i) control PBMCs and (ii) RE PBMCs, showing simultaneous electrographic seizures (red circles) in the hippocampus and cortex; (iii) continuous EEC traces were recorded in an RE-NSG mouse and are displayed as a bipolar montage. Data are representative of 17 or more animals per group. Scale bars: 1 $\mathrm{mV}, 10$ seconds ( $\mathrm{i}$ and $\mathrm{ii}$ ); 1 second (iii). (C) Percentage of NSG animals that developed clinical and electrographic seizures following engraftment with PBMCs from RE patients ( $n=7$ patients and 19 animals) or controls ( $n=5$ donors and 17 animals). ND, not detected. ${ }^{* *} P<0.001$, by $\chi^{2}$ test. (D) Kinetics of human and mouse immune cell infiltration into the CNS of NSC animals engrafted with PBMCs from RE patients (black circles) or control donors (white circles). Data shown represent the mean \pm SEM of absolute numbers of $\mathrm{mCD} 45^{+}$, hCD45 ${ }^{+}, \mathrm{hCD}^{+}$, and hCD8 ${ }^{+}$cells found in the brains of NSG animals between weeks 1 and 5 after transfer. $n=4-6$ animals per time point per group. ${ }^{*} P<0.05$, by unpaired, 2-tailed Student's $t$ test with Holm-Sidak correction.
Although Iba- $1^{+}$cells were infrequently observed in the brains of control-NSG mice, they were readily detected throughout the parenchyma of RE-NSG mice, occasionally forming small clusters of cells in proximity to $\mathrm{NeuN}^{+}$neurons (Supplemental Figure 3A). We also found evidence of focal neural damage in the brains of RE-NSG animals as shown by an increased immunoreactivity to amyloid precursor protein (APP) relative to the brains of control animals (Figure 3C). In addition, we detected a higher number of apoptotic cells (as assessed by TUNEL staining) that coexpressed the neuronal marker NeuN, predominantly in the brainstem, cerebellum, hemispheric gray matter, and hippocampal structures of RE-NSG mice (Figure 3D). To determine whether infiltrating human immune cells were directly responsible for inducing neuro- nal cell apoptosis in the brains of RE-NSG mice, we performed triple immunofluorescence staining for hCD $45^{+}, \mathrm{NeuN}$, and cleaved caspase- 3 and found infiltrating hCD $45^{+}$cells in direct apposition to neurons undergoing apoptosis (Figure 3E). The damage to neurons was probably mediated by cytotoxic $\mathrm{CD} 8^{+} \mathrm{T}$ lymphocytes, which had aggregated in perivascular cuffs or invaded the cerebral parenchyma in RE-NSG mice (Supplemental Figure 3B). Occasionally, hCD $45^{+}$cells were also found to be closely associated with cells displaying dual immunoreactivity for cleaved caspase-3 and for the microglial marker isolectin GS-B4 (IB4) (Figure 3E). In contrast, while hCD $45^{+}$cells were also detected in proximity to GFAPexpressing astrocytes, these were not immunopositive for activated caspase-3 (Supplemental Figure 3C). 
A
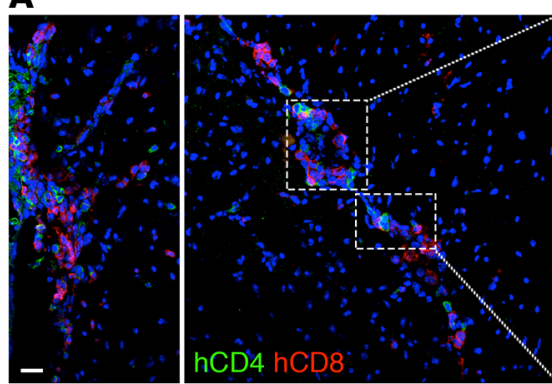

C
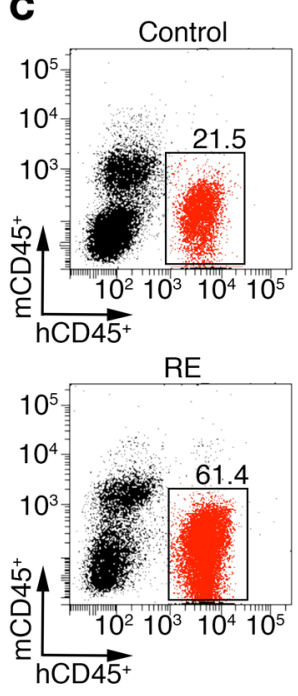

E

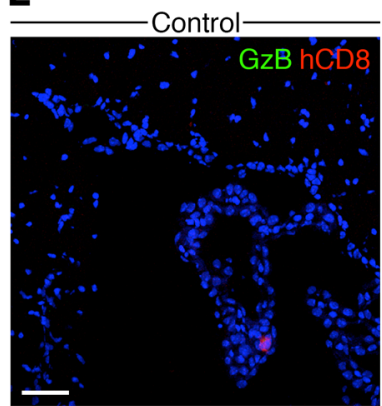

Gated on hCD4+

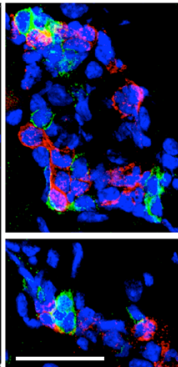

B

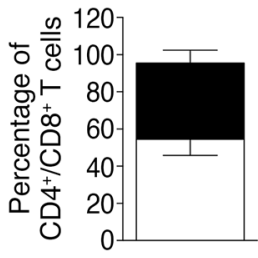

D

RE donors

Control donors
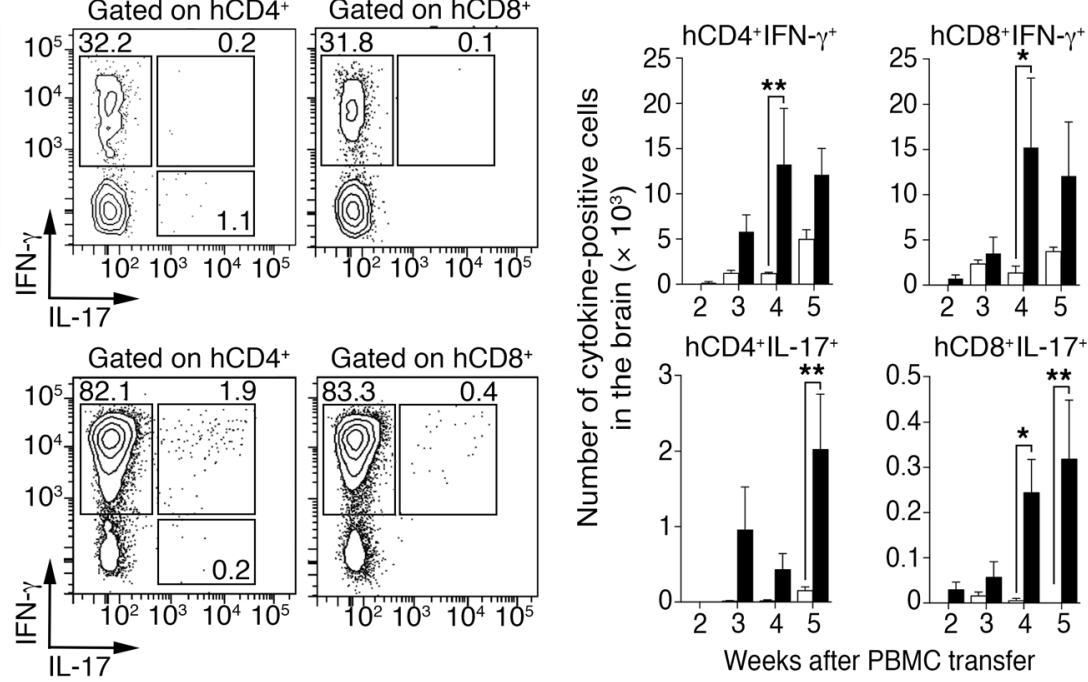

$\mathbf{F}$
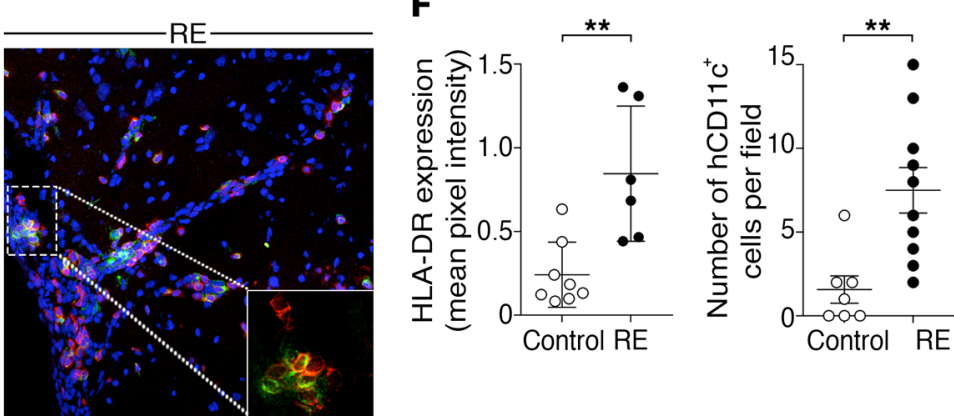

Figure 2. Cytokine production by hCD4 ${ }^{+}$and hCD8 ${ }^{+}$T lymphocytes infiltrating the CNS of control-NSG and RE-NSG animals. (A) Double immunofluorescence labeling for hCD4+ (green) and hCD8 ${ }^{+}$(red) T lymphocytes in the brains of RE-NSG mice 5 weeks after transfer. Scale bars: $30 \mu \mathrm{m}$. (B) Percentage of $\mathrm{hCD4}^{+}$(clear bar) and hCD8 ${ }^{+}$(solid bar) T lymphocytes in the brains of RE-NSG mice, as assessed by flow cytometry. Data represent the mean \pm SEM. $n=6$ mice. (C) Representative FACS dot plot and (D) kinetics of CNS infiltration by hCD4+ or hCD8 ${ }^{+}$cells expressing IFN- $\gamma$ and IL-17 in NSC animals engrafted with PBMCs from RE patients (black bars) or control donors (white bars). Data represent the mean \pm SEM of absolute numbers of immune cells. $n=4-6$ animals per time point and 2-3 human PBMC donors. ${ }^{*} P<0.05$ and ${ }^{* *} P<0.01$, by unpaired, 2-tailed Student's $t$ test with Holm-Sidak correction. (E) Confocal photomicrographs showing staining for hCD8 (red) and granzyme B (GzB, green) in the brains of control-NSG or RE-NSG mice. Images are representative of 7 fields from 6 sections obtained from 3 animals per group. Scale bar: $30 \mu \mathrm{m}$. Inset shows $\times 2$ magnification of the indicated area. (F) Fluorescence intensity for HLA-DR and quantification of hCD11c cells in the CNS of control-NSG and RE-NSG mice. Data represent the mean \pm SEM and are representative of 6 to 10 fields taken out of 6 sections from 3 animals per group. ${ }^{* *} P<0.01$, by unpaired, 2-tailed Student's $t$ test.

a4 integrin blockade reduces lymphocyte recruitment into the brains of RE-NSG mice. To validate the use of this experimental model for the development of therapies to treat RE, NSG animals were injected with PBMCs from 5 patients with RE, followed immediately by weekly administration of IVIGs, an immunotherapy that has proven beneficial in some patients with $\operatorname{RE}(4,6,31)$, or administration of PBS. EEG monitoring revealed that 12 of $13(92 \%)$ NSG animals that had received PBS developed seizures as compared with 4 of $12(25 \%)$ mice injected with IVIGs (Supplemental Figure 4A). We also detected fewer $\mathrm{hCD} 45^{+}$cells, with a switch in the $\mathrm{hCD} 4^{+} / \mathrm{hCD} 8^{+}$ratio (Supplemental Figure 4B) and a reduction of GFAP-reactive astrocytes in the CNS of animals that received IVIGs, as assessed by histology (Supplemental Figure 4C). 
A

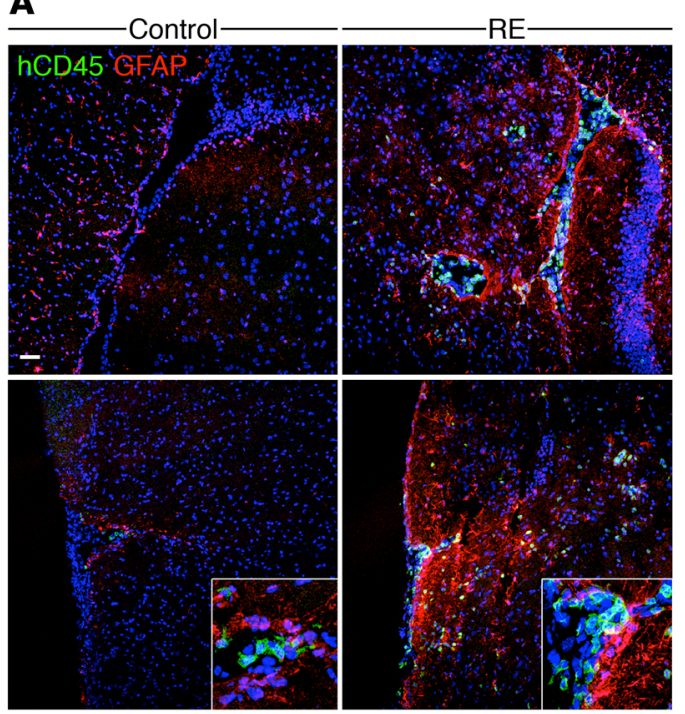

C

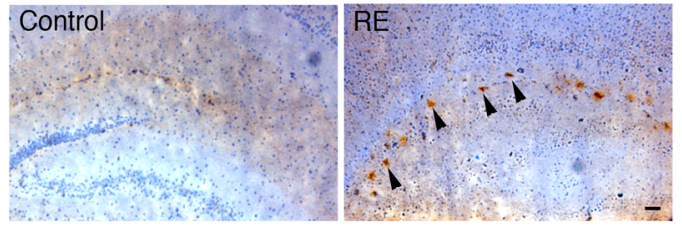

E
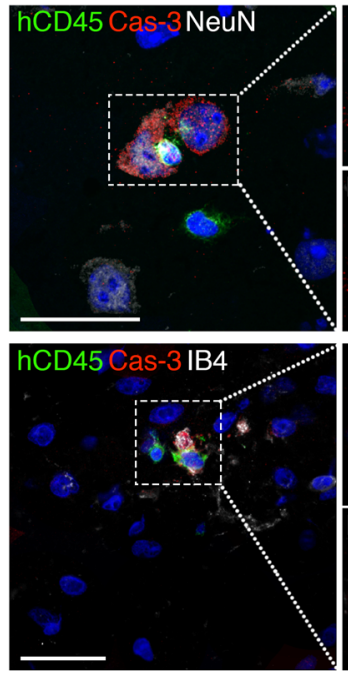

B

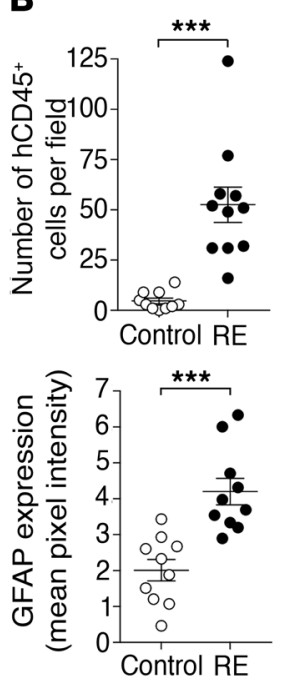

D

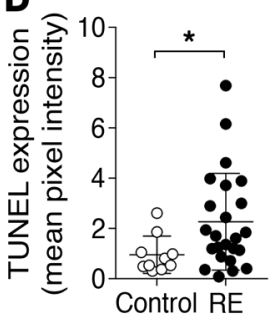

Figure 3. Neuropathology in RE-NSG animals. (A) Confocal photomicrograph showing GFAP (red) and hCD45 (green) in the CNS of control-NSC and RE-NSG mice 5 weeks after transfer. Scale bar: $30 \mu \mathrm{m}$; insets $\times 3$ magnification. (B) Quantification of hCD45+ cells and staining intensity for GFAP in the CNS of control-NSG and RE-NSG mice. Data represent the mean \pm SEM of 10 or more fields from 6 sections obtained from 3 animals per group. ${ }^{* *} P<0.001$, by unpaired, 2 -tailed Student's $t$ test. (C) Immunohistoperoxidase staining for APP in the hippocampal gyrus of control-NSG and RE-NSC mice 6 weeks after transfer. Arrowheads indicate $\mathrm{APP}^{+}$neuronal cells. Scale bar: $30 \mu \mathrm{m}$. (D) Quantification of cell death marker TUNEL in the CNS of control-NSC and RE-NSG mice 6 weeks after transfer. Data are expressed as the mean \pm SEM of 10 to 20 fields from 6 sections obtained from 2 animals per group. ${ }^{*} P<0.05$, by unpaired, 2-tailed Student's $t$ test. (E) Immunofluorescence staining for hCD45 (green), cleaved caspase-3 (Cas-3, red), and NeuN (upper panels, white) or IB4 (lower panels, white) in the brains of RE-NSG mice. DAPI (blue) was used for nuclear staining. Images are representative of 7 fields from 3 sections obtained from 3 RE-NSG mice. Scale bars: 20 $\mu \mathrm{m}$. The two upper left inset frames show $\times 1.33$ magnification of the dashed boxed area. All other insets are magnified $\times 2$.

therapeutic regimens induced a shift in the ratio of hCD4 and hCD8 T lymphocytes recruited to the CNS (Figure 4B). $\alpha 4$ integrin blockade also reduced the number of hCD $45^{+}$cells, GFAP-reactive astrocytes, HLA-DR ${ }^{+}$cells, and granzyme $\mathrm{B}^{+} \mathrm{hCD} 8^{+} \mathrm{T}$ lymphocytes when used therapeutically (Figure $4 \mathrm{C}$ ) or prophylactically (Figure 5, A-D). Video EEG monitoring revealed the absence of interictal and ictal manifestations in animals that were given anti- $\alpha 4$ antibody (0 of 6) prophylactically. In comparison, 5 of 6 animals (83\%) injected with isotype control developed seizures (Figure $5 \mathrm{E}$ ). Despite the significant reduction in immune cell infiltration when treatment was given therapeutically, seizure frequency and duration remained unaffected, suggesting that once disease is established, the reduction of $\mathrm{T}$ cell infiltration into the brain is not sufficient by itself to decrease the frequency of seizures and that additional antiepileptic treatments are required.

\section{Discussion}

Rare diseases, especially those like $\mathrm{RE}$ that affect pediatric populations, pose unique challenges for the development of therapies and for pathophysiological

Given the efficacy of anti-very late antigen-4 (anti-VLA-4) therapy for idiopathic CNS inflammation (i.e., multiple sclerosis) (32), we sought to evaluate whether blocking $\alpha 4$ integrin could alleviate the clinical and pathological signs of RE using our model. RE-NSG animals were given $\alpha 4$ integrin-blocking antibody or an isotype control in either a prophylactic (7 days after transfer) or therapeutic (after first seizures, week 4) setting. FACS analyses revealed a reduction in the number of infiltrating $\mathrm{hCD} 45^{+}$, $\mathrm{hCD}^{+}, \mathrm{hCD}^{+} \mathrm{T}$ lymphocytes as well as reduction of IFN- $\gamma-$ and IL-17-producing hCD $8^{+}$and $\mathrm{hCD} 4^{+} \mathrm{T}$ lymphocytes in the CNS of RE-NSG animals injected with anti- $\alpha 4$ integrin-neutralizing antibody, either prophylactically or therapeutically, as compared with isotype-treated RE-NSG mice (Figure 4A). Both prophylactic and studies. In recognition of this, we generated a humanized mouse model that reproduces the clinical and pathological features of RE by injecting patients' PBMCs into NSG mice. A similar approach was used to create models of other immune-mediated diseases such as idiopathic nephrotic syndrome, asthma, and systemic lupus (33-36). Previous attempts to induce RE by intracerebral injection of CNS biopsy material into primates, mice, and guinea pigs were unsuccessful (11), and the presence of autoantibodies against glutamate receptor 3 (GluR3), acetylcholine, NMDA, or mammalian uncoordinated-18 protein (MUNC18) has not been a consistent finding in the blood or brains of patients with RE (37-39).

Our data show that PBMCs from RE patients can directly induce seizures and pathological changes typical of $\mathrm{RE}$ when 
A

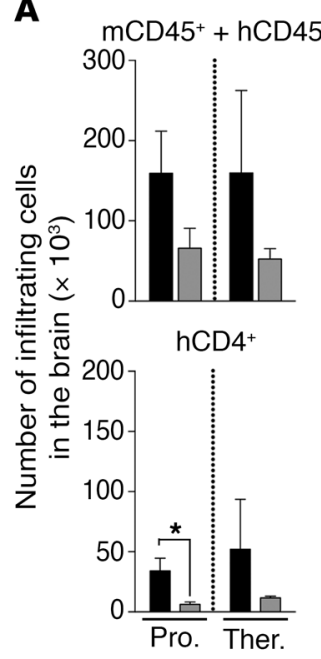

B

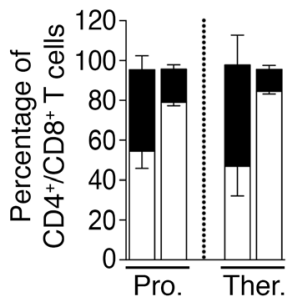

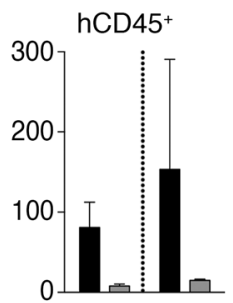

$\mathrm{hCD}^{+}$

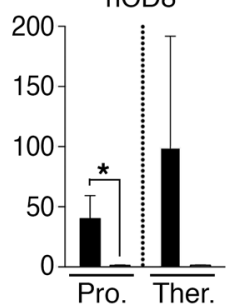

C
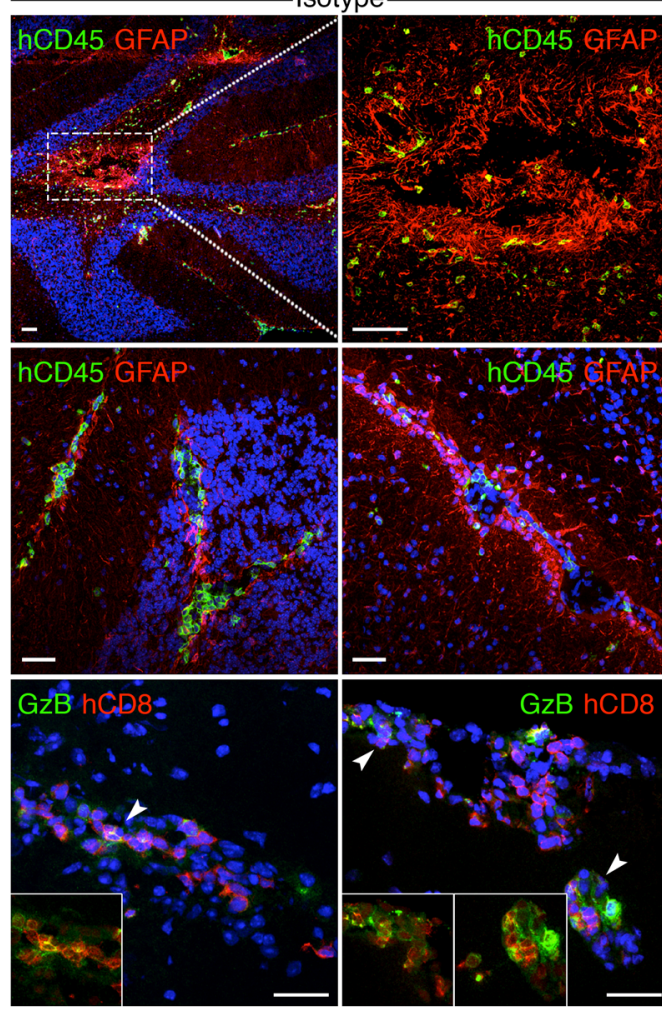
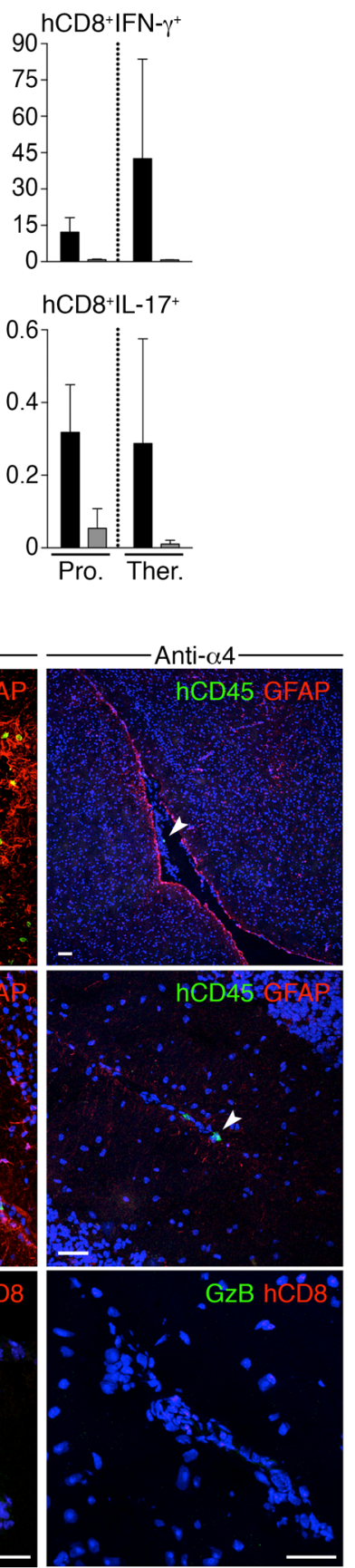

Figure 4. Effect of $\alpha 4$ integrin blockade on the clinical and immunological parameters of RE in NSG mice. (A) FACS analyses showing absolute numbers of mCD45 $45^{+}$cells and hCD45 ${ }^{+}$, hCD4 ${ }^{+}$ and hCD8 $8^{+}$cells expressing IFN- $\gamma$ or IL-17 in the CNS of RE-NSC mice treated with isotype control (black bars) or anti- $\alpha 4-$ neutralizing antibodies (gray bars), either in a prophylactic (Pro.) or therapeutic (Ther.) setting. Data represent the mean \pm SEM.

${ }^{*} P<0.05$, by unpaired, 2 -tailed Student's $t$ test. (B) Percentage of hCD4 ${ }^{+}$(white bars) and hCD8 ${ }^{+}$ (black bars) T lymphocytes in the brains of RE-NSC mice treated with isotype control (left bars) or anti- $\alpha 4$-neutralizing antibodies (right bars), either in a prophylactic or therapeutic setting. Data represent the mean \pm SEM. $n=2-6$ mice. (C) Confocal photomicrographs showing GFAP, hCD45, hCD8, and GzB staining in the brains of RE-NSC mice treated with isotype control or anti- $\alpha 4$-neutralizing antibodies in a therapeutic setting. Images are representative of 6 to 10 fields from 4 sections obtained from 2 animals per group. Scale bars: $30 \mu \mathrm{m}$. engrafted into NSG mice, therefore inferring that the development of seizures in RE is an intrinsic property of patients' immune cells and providing convincing evidence of the encephalitogenicity of RE patients' mononuclear cells. We also reveal the immunological nature of the disease by providing a thorough characterization of the kinetics of immune cell infiltration into the brain, in particular the contribution of CD4 and CD8 T lymphocytes and MHC class II antigen-presenting cells to the development of the disease in mice.

One notable difference between our experimental model and the human disease is the bilateral hemispheric involvement in NSG mice, whereas for the large majority of RE patients, the disease remains unihemispheric in nature. Some have suggested that the unilaterality of the disease in humans could be secondary to focal cortical dysplasia or to a subtle congenital malformation during brain development. This hypothesis is supported by cases of dual pathology, as assessed by neurohistopathological examination of surgical specimens from 7 patients with a confirmed RE diagnosis and concomitant cortical dysplasia (40). Our model suggests that, in the absence of dysplasia in mice, patients' PBMCs can intrinsically induce seizures and immune cell infiltration into both hemispheres.

One drawback associated with the use of NSG mice is the onset of GVHD within weeks after human PBMC transfer, a phenomenon that could potentially interfere with the pathophysiological process of RE. To prevent the occurrence of GVHD and maximize the applicability of the experimental RE model, we injected NSG mice with a lower dose of human PBMCs $\left(1 \times 10^{6}\right.$ cells com- 
A

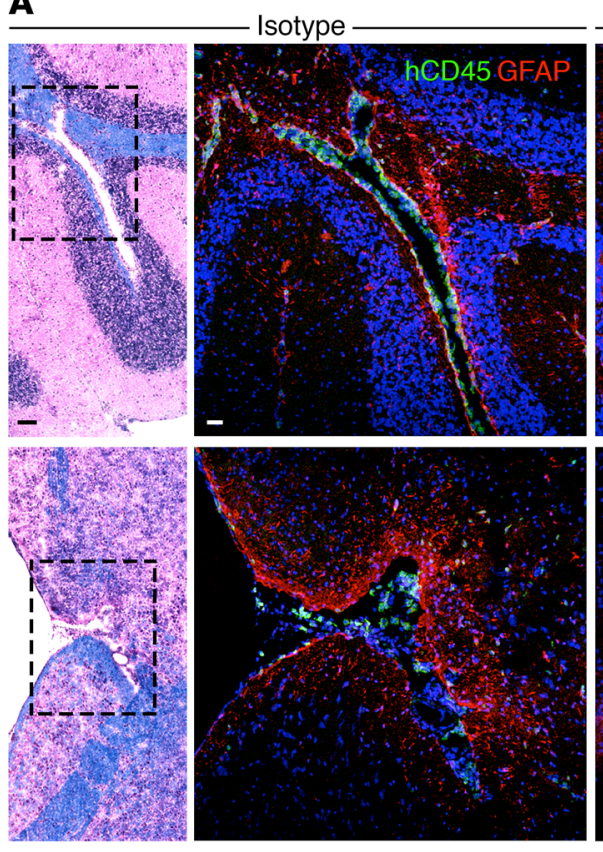

c

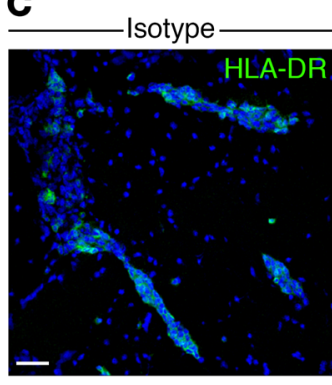

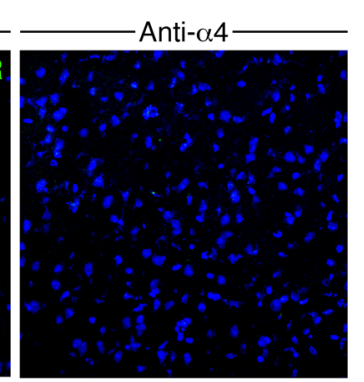
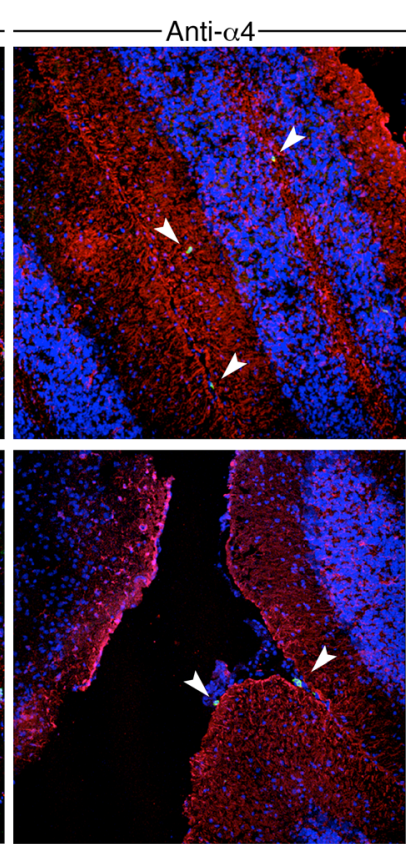

D

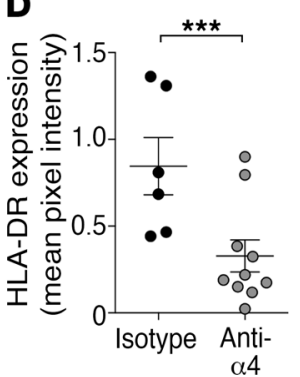

B

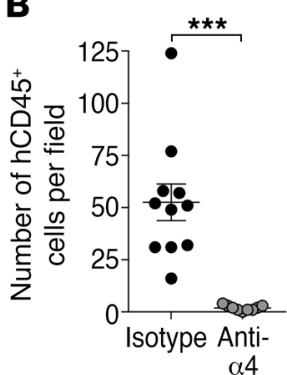

$\alpha 4$

$\mathbf{E}$

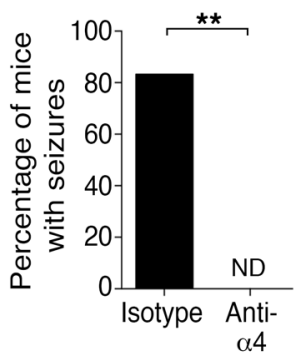

Figure 5. Effect of prophylactic treatment with anti- $\alpha 4$ integrin antibody on the development of disease in RE-NSG mice. RE-NSG mice were treated with isotype control (black circles) or anti- $\alpha 4$ antibody (gray circles) in a prophylactic setting. The effect of $\alpha 4$ integrin blockade was assessed for (A and $\mathbf{B}$ ) the infiltration of hCD45+ and GFAP in the brains of RE-NSG mice, (C and $\mathbf{D}$ ) the expression of HLA-DR, and (E) the occurrence of seizures. Arrowheads point to the few hCD45 cells detected in the brains of RE-SNG mice treated with anti- $\alpha 4$ integrin antibody. Images are representative of 6 to 11 fields from 4 sections obtained from 2 to 4 animals per group. Scale bars: $30 \mu \mathrm{m}$. Data shown in $\mathbf{E}$ are expressed as the mean \pm SEM. ${ }^{* *} P<0.01$ and ${ }^{* * *} P<0.001$, by unpaired, 2-tailed Student's $t$ test (B and D) and $\chi^{2}$ test (E). pared with the dose of $10 \times 10^{6}$ cells typically used; ref. 30 ). In our experimental setting, mice did not show signs of GVHD, and all maintained a score of 0 on the GVHD scale (41) over the 50-day observation period following PBMC transfer (at a dose of $1 \times 10^{6}$ immune cells). In the future, this limitation could be circumvented by the use of NSG mice devoid of murine MHC molecules, which can be engrafted by human PBMCs, without inducing any GVHD. Further studies of these mice could reveal whether stimulation of human $\mathrm{T}$ cells by murine MHC is required for the development of $\mathrm{RE}$ and will provide insights into the mechanism underlying seizure development and immune cell infiltration into the brain.

In addition, we found that anti-VLA-4 therapy, which is known to block the entry of immune cells into target organs (42-44), was highly effective at reducing pathological determinants of disease when given either prophylactically or therapeutically. These observations suggest that the recruitment of T lymphocytes into the CNS is directly involved in the development of seizures in $\mathrm{RE}$ and that the epileptic syndrome is not solely induced by a cytokine storm in the peripheral blood. However, the lack of efficacy of anti-VLA-4 in controlling seizures once they have begun suggests that preexisting brain lesions, presumably resulting from a first wave of $\mathrm{T}$ cell infiltration into the CNS, can trigger seizures even when the inflammatory process has resolved, thus highlighting the challenge in treating seizures in humans once the disease is established. These findings also emphasize the need to perform immunological intervention early in the course of the disease. Alternatively, it may be reasonable to consider combination therapy with coadministration of anti-VLA-4 and antiepileptic medications in order to reduce seizure frequency, as was successfully achieved in a single case report (45).

$\mathrm{RE}$ is a rare disease that may be difficult to diagnose in the early stages, or when MRI findings are not specific or brain biopsies are difficult to perform. The occurrence of seizures in mice 3 to 4 weeks after receiving patients' PBMCs could be used as a model to confirm a diagnosis of RE. This model could also be used to evaluate the efficacy of various therapies in a personalized, patient-centric manner, tailoring treatment to the individual's response in terms of controlling seizures and reducing brain infiltration.

\section{Methods}

$R E$ patients and control subjects. Blood was collected from 5 control subjects and 7 RE patients. Their characteristics are listed in Supplemental Table 1. All patients (4 males and 3 females between the ages of 8 and 51 years; median age of 11 years) presented with intractable focal seizures that were classified as partial seizures and evolved toward EPC and hemiparesis. Only 2 patients experienced secondarily generalized seizures. At the time of PBMC harvesting, 5 of the RE patients were in the acute stage of the disorder (stage 2), while the others were in its residual (chronic inactive) stage (stage 3). EEG showed lateral- 
ized epileptic activity involving the centro-temporal and/or frontotemporal regions in all of these patients. MRI results were abnormal in all patients, with left-sided involvement in 5 patients and right-sided involvement in 2 patients. None of the patients had evidence of bilateral disease, and 5 patients had biopsy/surgery-proven RE. All patients were negative for circulating autoantibodies against NMDA or other voltage-gated neuronal/glial antigens.

The control subjects included 2 males and 3 females, aged 8-26 years (median age of 11 years). There were 4 healthy control subjects and 1 child with refractory temporal lobe epilepsy.

$P B M C$ isolation and transfer. PBMCs from patients or control subjects were isolated under Ficoll-Hypaque gradient centrifugation. Six- to twelve-week-old mice were injected with either freshly isolated PBMCs or thawed PBMCs that had been frozen in 10\% DMSO. Prior to the injection, 7-9 NSG mice (The Jackson Laboratory) were sublethally irradiated at 2 to 3 Gy using a Gammacell 1000 (MDS Nordion) or a Faxitron CP-160 (Faxitron Bioptics). Five to six hours after irradiation, the mice were injected i.p. with $1 \times 10^{6}$ PBMCs. In some experiments, PBMCs were irradiated at $54 \mathrm{~Gy}$ using a Cobalt- 60 irradiator prior to injection to promote senescence and inactivation in vivo. At least 3 mice were injected with PBMCs from each human blood sample.

IVIG treatment and anti- $\alpha 4$ integrin blockade. RE-NSG mice were injected i.p. once a week, starting on day 0 after reconstitution until sacrifice, with $50 \mathrm{mg}$ IVIG (Gamunex, Grifols Therapeutics) or PBS. For the anti- $\alpha 4$ integrin blockade experiment, RE-NSG mice were injected i.p. twice weekly with $75 \mu \mathrm{g}$ human integrin $\alpha 4$ monoclonal antibody (clone 2B4) or with mouse IgG1 isotype control (both from R\&D Systems). Prophylactic treatment was started on day 7 after PBMC transfer and lasted until sacrifice. Therapeutic treatment was initiated after the onset of seizures, at week 4 after PBMC transfer.

Detection of seizures by video EEG recording. Mice were monitored by continuous video EEG recordings, with electrodes implanted bilaterally into the somatosensory cortex and the limbic system to detect seizures. EEG recordings started between days 14 and 21 after PBMC transfer. Specific stereotactic insertion of electrodes was performed surgically under constant general anesthesia with isoflurane. Briefly, a stainless steel bipolar electrode (Plastics-1 Inc.) was positioned in the right limbic system at the following coordinates with reference to the bregma: anteroposterior $(\mathrm{AP})=-1.5$, lateral $(\mathrm{L})=-3.6$, and dorsal-ventral $(\mathrm{DV})$ $=8.0$. Prior to implantation, one of the contacts had been sectioned to remain in the overlying cortex. EEGs were recorded with a Stellate Systems 32-channel video EEG recording unit, enabling the recording and visual monitoring of 8 mice at a time. Mice were monitored 4 hours a day over a 30-day period for electrical and clinical seizures. Two separate observers (blinded to the treatment groups) reviewed the EEGs.

Engraftment. The presence of circulating human cells was evaluated weekly in $100 \mu \mathrm{l}$ peripheral blood harvested through the saphenous vein and collected in heparinized tubes. The amount of cells was assessed by FACS using monoclonal antibodies directed against hCD45 (clone HI30), mCD45 (clone 30-F11), hCD3 (clone UCHT1), hCD19 (clone HIB19), and hCD56 (clone B159) (all from BD Biosciences). Acquisition was done on a FACSAria or FACS LSRFortessa system (BD Biosciences). In some experiments, bone marrow, thymus, spleen, and brain were harvested when the mice were sacrificed.

Flow cytometry. Animals were sacrificed at given time points following PBMC transfer and perfused with cold PBS via transcardiac puncture. The spleen and 1 hemisphere of the brain were processed for cell isolation and flow cytometric analysis as previously described (46). Splenic and brain cells were stimulated for 6 hours with PMA (20 ng/ $\mathrm{ml})$ and ionomycin $(1 \mu \mathrm{g} / \mathrm{ml})$ in the presence of brefeldin A $(2 \mu \mathrm{g} / \mathrm{ml})$ (all from Sigma-Aldrich). Cells were first stained for the surface antigens mCD45 (clone 30-F11), hCD45 (clone HI30), hCD4 (clone SK3), and hCD8 (clone HIT8a) (all from BD Biosciences) and then fixed in $4 \%(\mathrm{w} / \mathrm{v})$ paraformaldehyde and permeabilized with $0.1 \%$ saponin. Intracellular cytokine staining was performed using antibodies specific for human IL-17 (eBioscience, clone eBio64CAP17) and IFN- $\gamma$ (BD Biosciences, clone B27). Appropriate fluorochrome-matched isotype antibodies were used to determine nonspecific background staining. Samples were acquired on a BD Biosciences LSR II Flow Cytometer at the Flow Cytometry Core Facility of CRCHUM and analyzed using BD FACSDiva software. Viable cells were counted in 1 hemisphere using trypan blue to estimate the absolute number of $\mathrm{mCD} 45^{+}$and hCD $45^{+}$ cells infiltrating the brain. The number of $\mathrm{hCD} 4^{+}, \mathrm{hCD} 8^{+}$, and IFN- $\gamma^{-}$ and IL-17-producing $\mathrm{T}$ lymphocytes in the brain was then determined by multiplying the percentage of lineage marker-positive cells detected by FACS and the total number of hCD $45^{+}$cells.

Immunohistostainings. Brains from control-NSG and RE-NSG mice were collected following rapid intracardiac PBS perfusion, and 1 of the hemispheres was snap-frozen in liquid nitrogen. Histopathological characterization was performed by luxol fast blue hematoxylineosin (LHE) staining as previously described (47). Sagittal cryosections (7- $\mu \mathrm{m}$-thick) were cut using a Leica CM3050S cryostat and mounted onto superfrost slides, fixed in $-20^{\circ} \mathrm{C}$ acetone for $10 \mathrm{~min}-$ utes, and hydrated in PBS. The Mouse On Mouse (M.O.M.) Kit (Vector Laboratories) was used when required to block endogenous mouse antibodies in the tissue section. Otherwise, nonspecific Ig binding was blocked with $10 \%$ donkey serum for 30 minutes. Sections were incubated overnight at $4^{\circ} \mathrm{C}$ or for 2 hours at room temperature with the following primary antibodies: mouse anti-hCD45 (BD Biosciences, clone HI30, dilution 1:30); mouse anti-HLA-DR (Dako, clone TAL.1B5, dilution 1:50); mouse anti-hCD11c (BD Biosciences, clone B-ly6, dilution 1:40); mouse anti-hCD19 (BD Biosciences, clone HIB19, dilution 1:70); rabbit anti-hCD8 (Vector Laboratories, clone SP16, dilution 1:50); mouse anti-human granzyme B (Thermo Fisher Scientific, clone GB12, 1:10); Cy3-conjugated mouse anti-GFAP (Sigma-Aldrich, clone G-A-5, dilution 1:1000); Alexa Fluor 647conjugated mouse anti-GFAP (BD Biosciences, clone 1B4, dilution 1:50); rabbit anti-Iba-1 (Wako, 019-19741, dilution 1:100); Alexa Fluor 647-conjugated isolectin GS-IB4 (Thermo Fisher Scientific, I32450, dilution 1:300); mouse biotinylated anti-NeuN (US Biological, clone $3 \mathrm{H} 412$, dilution 1:200); and rabbit anti-human cleaved caspase-3 (Cell Signaling Technology, 9661, dilution 1:400). Sections were then incubated for 40 minutes at room temperature with the appropriate secondary antibodies: Alexa Fluor 488-conjugated donkey anti-mouse (Jackson ImmunoResearch, 715-545-150, dilution 1:400); Alexa Fluor 488-conjugated donkey anti-rabbit (Thermo Fisher Scientific, A21206, dilution 1:400); rhodamine red X-conjugated (RRXconjugated) donkey anti-rabbit (Jackson ImmunoResearch, 711-295152, dilution 1:400); Cy3-labeled donkey anti-mouse (Jackson ImmunoResearch, 715-165-150, dilution 1:200); Cy3-conjugated streptavidin (Jackson ImmunoResearch, 016-160-084, dilution 1:1,500); and Alexa Fluor 647-conjugated streptavidin (Thermo Fisher Scientific, S32357, dilution 1:500). Negative controls were performed, omitting the primary antibody. TO-PRO-3 (Thermo Fisher Scientific, dilution 
1:300) or DAPI (Sigma-Aldrich, dilution 1:1,000) was used for nuclear staining. Fluorescence acquisition was done on a Leica SP5 confocal microscope at the CRCHUM Cell Imaging Core Facility. Staining for terminal TUNEL labeling was performed using the TMR Red In Situ Cell Death Detection Kit (Roche).

To determine axonal damage, control-NSG and RE-NSG brain cryosections were fixed in cold acetone for 10 minutes at $-20^{\circ} \mathrm{C}$ and then transferred to ethanol for 5 minutes at $-20^{\circ} \mathrm{C}$. Sections were washed in PBS, treated with $0.03 \%$ hydrogen peroxide for 10 minutes, washed again with PBS containing 0.05\% Tween-20, and blocked at room temperature for 30 minutes with $10 \%$ goat serum. Sections were then incubated for 1 hour at room temperature with rabbit antiamyloid precursor protein (anti-APP) (Sigma-Aldrich, A8967, dilution 1:100). Afterwards, slides were washed 7 times in PBS/Tween-20. Goat anti-rabbit HRP-labeled antibody (Jackson ImmunoResearch, 111-035-144, dilution 1:400) was incubated at room temperature for 30 minutes, and the immunoperoxidase reaction was developed using DAB (Dako) as a chromogen. Sections were counterstained with hematoxylin for 20 seconds, differentiated for 1 second with acid alcohol, and dehydrated in 70\% ethanol, 95\% ethanol, absolute ethanol, and $50 \%$ ethanol in toluene and in 3 baths of toluene (1 minute each). Sections were mounted with Entellan (Sigma-Aldrich) and then imaged and processed as described above.

Quantitative analyses. hCD 45 and hCD11c cells were counted in micrographs taken of 7 to 11 random fields in cryosections of the brains of control-NSG and RE-NSG mice. Mean pixel intensity was evaluated using Image software $(\mathrm{NIH})$ in confocal images of control-NSG and RE-NSG brains stained for HLA-DR, GFAP, and TUNEL. All evaluations were performed independently by at least 2 investigators.

Statistics. Statistical analyses were performed using GraphPad Prism 6.0 (GraphPad Software). Data are presented as the mean \pm SEM. Statistical significance was determined by unpaired, 2-tailed Student's $t$ test, except for the graphs of the percentage of mice with seizures, which were analyzed using a $\chi^{2}$ test. Only $P$ values of less than 0.05 were considered statistically significant.

Study approval. Studies involving human subjects were approved by the CHU Sainte-Justine Ethics Committee (Montreal, Canada; approval number 2602). Written informed consent was obtained from the healthy volunteers and from RE patients and/or their legal guardians. All animal experiments were approved by the CHU SainteJustine Research Ethics Committee and performed in compliance with the Comité Institutionnel des Bonnes Pratiques Animales en Recherche (CIBPAR) (approval number 528).

\section{Author contributions}

$\mathrm{HK}, \mathrm{LC}, \mathrm{FF}, \mathrm{AP}$, and EH generated the hypotheses, conceptualized the study, and wrote the manuscript. KB carried out animal work and coordinated the project. CMB, NTS, and SD performed surgeries in mice for electrode implantation. LC and NTS analyzed EEG recordings. HK designed and performed the flow cytometric experiments and histopathology studies. JIA and CLP assisted with immunostainings and confocal microscopy. LC, DH, MJL, RMR, DKN, CCW, GLH, HPG, JRM, and MBC performed clinical assessment of the patients and collected samples. All authors reviewed and approved the manuscript.

\section{Acknowledgments}

We gratefully acknowledge Simone Terouz and Janet Laganière (from the CRCHUM, Montreal, Quebec, Canada) for their technical assistance. We also thank Aurélie Cléret-Buhot from the CRCHUM Cell Imaging Core Facility. This work was supported by a grant from the Canadian Institutes of Health Research (CIHR; 201203MOP-274421 to LC, EH, and AP) and by the RE Children's Project who provided bridge funding. AP holds a Senior Canada Research Chair in Multiple Sclerosis.

Address correspondence to: Alexandre Prat, CHUM Research Center, 900 Saint Denis Street, Pavillion R, Montreal, Quebec, Canada, H2X 0A9. Phone: 514.890.8000 ext. 24734; Email: a.prat@umontreal.ca. Or to: Elie Haddad, CHU Sainte-Justine Research Center, 3175 Chemin de la Côte Sainte-Catherine, Montreal, Quebec, Canada, H3T 1C5. Phone: 514.345.4713; Email: elie.haddad@umontreal.ca.

JRM's present address is: Department of Pediatrics, Division of Pediatric Neurology, Nationwide Children's Hospital, Ohio State University, Columbus, Ohio, USA.
1. Rasmussen T, Olszewski J, Lloydsmith D. Focal seizures due to chronic localized encephalitis. Neurology. 1958;8(6):435-445.

2. Bien CG, et al. Pathogenesis, diagnosis and treatment of Rasmussen encephalitis: a European consensus statement. Brain. 2005;128(Pt 3):454-471.

3. Bien CG, Schramm J. Treatment of Rasmussen encephalitis half a century after its initial description: promising prospects and a dilemma. Epilepsy Res. 2009;86(2-3):101-112.

4. Leach JP, Chadwick DW, Miles JB, Hart IK. Improvement in adult-onset Rasmussen's encephalitis with long-term immunomodulatory therapy. Neurology. 1999;52(4):738-742.

5. Bien CG, Gleissner U, Sassen R, Widman G, Urbach H, Elger CE. An open study of tacrolimus therapy in Rasmussen encephalitis. Neurology. 2004;62(11):2106-2109.

6. Granata T, et al. Experience with immunomodulatory treatments in Rasmussen's encephalitis.
Neurology. 2003;61(12):1807-1810.

7. Bien CG, et al. Rasmussen encephalitis: incidence and course under randomized therapy with tacrolimus or intravenous immunoglobulins. Epilepsia. 2013;54(3):543-550.

8. Takahashi Y, et al. Immunomodulatory therapy versus surgery for Rasmussen syndrome in early childhood. Brain Dev. 2013;35(8):778-785.

9. Thilo B, et al. A case of Rasmussen encephalitis treated with rituximab. Nat Rev Neurol. 2009;5(8):458-462.

10. Hart Y. Rasmussen's encephalitis. Epileptic Disord. 2004;6(3):133-144.

11. Asher DM, Gajdusek DC. Virologic studies in chronic encephalitis. In: Andermann F, ed. Chronic encephalitis and epilepsy: Rasmussen's Syndrome. Stoneham MA:ButterworthHeinmann;1991:147-158.

12. Walter GF, Renella RR. Epstein-Barr virus in brain and Rasmussen's encephalitis. Lancet.
1989;1(8632):279-280.

13. Power C, Poland SD, Blume WT, Girvin JP, Rice GP. Cytomegalovirus and Rasmussen's encephalitis. Lancet. 1990;336(8726):1282-1284.

14. Jay V, et al. Chronic encephalitis and epilepsy (Rasmussen's encephalitis): detection of cytomegalovirus and herpes simplex virus 1 by the polymerase chain reaction and in situ hybridization. Neurology. 1995;45(1):108-117.

15. Andrews JM, Thompson JA, Pysher TJ, Walker ML, Hammond ME. Chronic encephalitis, epilepsy, and cerebrovascular immune complex deposits. Ann Neurol. 1990;28(1):88-90.

16. Whitney KD, Andrews PI, McNamara JO. Immunoglobulin $\mathrm{G}$ and complement immunoreactivity in the cerebral cortex of patients with Rasmussen's encephalitis. Neurology. 1999;53(4):699-708.

17. Rogers SW, et al. Autoantibodies to glutamate receptor GluR3 in Rasmussen's encephalitis. Science. 1994;265(5172):648-651. 
18. Andrews PI, Dichter MA, Berkovic SF, Newton MR, McNamara JO. Plasmapheresis in Rasmussen's encephalitis. Neurology. 1996;46(1):242-246.

19. Twyman RE, Gahring LC, Spiess J, Rogers SW. Glutamate receptor antibodies activate a subset of receptors and reveal an agonist binding site. Neuron. 1995;14(4):755-762.

20. Levite M, Fleidervish IA, Schwarz A, Pelled D, Futerman AH. Autoantibodies to the glutamate receptor kill neurons via activation of the receptor ion channel. J Autoimmun. 1999;13(1):61-72.

21. Whitney KD, McNamara JO. GluR3 autoantibodies destroy neural cells in a complement-dependent manner modulated by complement regulatory proteins. J Neurosci. 2000;20(19):7307-7316.

22. Levite M, Hermelin A. Autoimmunity to the glutamate receptor in mice--a model for Rasmussen's encephalitis? J Autoimmun. 1999;13(1):73-82.

23. Bien CG, et al. Destruction of neurons by cytotoxic T cells: a new pathogenic mechanism in Rasmussen's encephalitis. Ann Neurol. 2002;51(3):311-318.

24. Bauer J, et al. Astrocytes are a specific immunological target in Rasmussen's encephalitis. Ann Neurol. 2007;62(1):67-80.

25. Varadkar S, et al. Rasmussen's encephalitis: clinical features, pathobiology, and treatment advances. Lancet Neurol. 2014;13(2):195-205.

26. Schwab N, et al. CD8+ T-cell clones dominate brain infiltrates in Rasmussen encephalitis and persist in the periphery. Brain. 2009;132(Pt 5):1236-1246.

27. Li Y, et al. Local-clonal expansion of infiltrating $\mathrm{T}$ lymphocytes in chronic encephalitis of Rasmussen. J Immunol. 1997;158(3):1428-1437.

28. Shultz LD, Brehm MA, Garcia-Martinez JV, Greiner DL. Humanized mice for immune system investigation: progress, promise and chal- lenges. Nat Rev Immunol. 2012;12(11):786-798.

29. Walsh NC, et al. Humanized Mouse Models of Clinical Disease. Annu Rev Pathol. 2017;12:187-215.

30. Gregoire-Gauthier J, et al. Use of immunoglobulins in the prevention of GvHD in a xenogeneic NOD/SCID/ $\gamma \mathrm{c}$ - mouse model. Bone Marrow Transplant. 2012;47(3):439-450.

31. Hart YM, et al. Medical treatment of Rasmussen's syndrome (chronic encephalitis and epilepsy): effect of high-dose steroids or immunoglobulins in 19 patients. Neurology. 1994;44(6):1030-1036.

32. Polman $\mathrm{CH}$, et al. A randomized, placebocontrolled trial of natalizumab for relapsing multiple sclerosis. N Engl J Med. 2006;354(9):899-910.

33. Kerre TC, et al. Adapted NOD/SCID model supports development of phenotypically and functionally mature $\mathrm{T}$ cells from human umbilical cord blood CD34(+) cells. Blood. 2002;99(5):1620-1626.

34. Sellier-Leclerc AL, et al. A humanized mouse model of idiopathic nephrotic syndrome suggests a pathogenic role for immature cells. J Am Soc Nephrol. 2007;18(10):2732-2739.

35. Andrade D, et al. Engraftment of peripheral blood mononuclear cells from systemic lupus erythematosus and antiphospholipid syndrome patient donors into BALB-RAG-2-/- IL-2R $\gamma$-/mice: a promising model for studying human disease. Arthritis Rheum. 2011;63(9):2764-2773.

36. Traggiai E, et al. Development of a human adaptive immune system in cord blood cell-transplanted mice. Science. 2004;304(5667):104-107.

37. Wiendl H, et al. GluR3 antibodies: prevalence in focal epilepsy but no specificity for Rasmussen's encephalitis. Neurology. 2001;57(8):1511-1514.

38. Watson R, et al. Absence of antibodies to glutamate receptor type 3 (GluR3) in Rasmussen encephalitis. Neurology. 2004;63(1):43-50.

39. Mantegazza R, et al. Antibodies against GluR3 peptides are not specific for Rasmussen's encephalitis but are also present in epilepsy patients with severe, early onset disease and intractable seizures. J Neuroimmunol. 2002;131(1-2):179-185.

40. Takei H, et al. Dual pathology in Rasmussen's encephalitis: a study of seven cases and review of the literature. Neuropathology. 2010;30(4):381-391.

41. Cooke KR, et al. An experimental model of idiopathic pneumonia syndrome after bone marrow transplantation: $\mathrm{I}$. The roles of minor $\mathrm{H}$ antigens and endotoxin. Blood. 1996;88(8):3230-3239.

42. Yednock TA, Cannon C, Fritz LC, SanchezMadrid F, Steinman L, Karin N. Prevention of experimental autoimmune encephalomyelitis by antibodies against alpha 4 beta 1 integrin. Nature. 1992;356(6364):63-66.

43. Podolsky DK, et al. Attenuation of colitis in the cotton-top tamarin by anti-alpha 4 integrin monoclonal antibody. J Clin Invest. 1993;92(1):372-380.

44. Sandborn WJ, Yednock TA. Novel approaches to treating inflammatory bowel disease: targeting alpha-4 integrin. Am J Gastroenterol. 2003;98(11):2372-2382.

45. Bittner S, Simon OJ, Göbel K, Bien CG, Meuth SG, Wiendl H. Rasmussen encephalitis treated with natalizumab. Neurology. 2013;81(4):395-397.

46. Ifergan I, et al. Role of Ninjurin-1 in the migration of myeloid cells to central nervous system inflammatory lesions. Ann Neurol. 2011;70(5):751-763.

47. Alvarez JI, et al. Focal disturbances in the bloodbrain barrier are associated with formation of neuroinflammatory lesions. Neurobiol Dis. 2015;74:14-24. 